\title{
La Relación de la Investigación Científica con la Motivación de Logro Académico en los Estudiantes de Postgrado de la Universidad Alas Peruanas
}

\section{The Relationship of Scientific Research with the Motivation of Academic Achievement in the Postgraduate Students of Alas Peruanas University}

\author{
Isabel Carhuamaca ${ }^{1}$
}

\section{RESUMEN}

El presente artículo tiene carácter investigativo y cómo objetivo es determinar la relación que existe entre: La investigación científica y la motivación de logro académico. Este trabajo es de tipo descriptivo y correlacional, y corresponde a un diseño no experimental, de corte transversal, para ello se aplica un cuestionario que incluyó ítems de ambas variables, para ello se usa la técnica de la encuesta. El instrumento es validado a través el alfa de cronbach para medir la confiabilidad. Se está utilizando el análisis de relaciones utiliza las correlaciones simples entre los puntajes de las dimensiones y su respectiva variable, luego se calcula el coeficiente de correlación de Spearman para cada dimensión, por ser la adecuada para este tipo de variables (ordinales) con ello se construye el modelo estructurado ecuación modelo siendo los siguientes resultados: La Investigación Científica se relaciona directa y significativamente a un nivel de confianza del 95\% con la motivación de logro académico según los Estudiantes de Postgrado Maestría en la Universidad Alas Peruanas Lima. Cuando Existe una buena motivación de logro académico se va a realizar una buena investigación científica por parte de los estudiantes.

Palabra Clave: Investigación Científica, Motivación de Logro Académico, Dimensión, Relación

\section{ABSTRACT}

The present article has investigative character and as objective it is to determine the relation that exists between: The scientific investigation and the motivation of academic achievement. This work is descriptive and correlational, and corresponds to a non-experimental, cross-sectional design, for which a questionnaire was applied that included items of both variables, for which the survey technique is used. The instrument is validated through the cronbach alpha to measure reliability. The relationship analysis uses the simple correlations between the scores of the dimensions and their respective variable, then the Spearman correlation coefficient is calculated for each dimension, being the appropriate for this type of variables (ordinal). Constructs the structured model model equation, with the following results: Scientific Research is directly and significantly related to a level of $95 \%$ confidence with the academic achievement motivation according to Postgraduate Students Master in Alas Peruanas University Lima. When there is a good academic achievement motivation is going to make a good scientific research by students.

Key Word: Scientific Research, Academic Achievement Motivation, Dimension, Relationship.

1. Mg. en Evaluación y Acreditación de la Calidad Educativa - Universidad Nacional Mayor de San Marcos E-mail: isbelcris7@hotmail.com 


\section{INTRODUCCIÓN}

La necesidad de responder con éxito a las demandas de una sociedad cada día más exigente y cambiante, han llevado a las organizaciones de Educación Universitaria a realizar grandes esfuerzos de innovación mejoramiento para lograr la calidad total en investigación científica.

El presente trabajo de investigación es importante porque responde a un problema Nacional, la falta de competitividad para la investigación. Es objetivo, porque es un problema real, evidente y se sustenta en las evaluaciones de Shangai y Stamford donde ninguna Universidad Peruana aparece entre las 500 primeras Universidades del mundo. Todo ello debido a la carencia de investigación y difusión del conocimiento, entre otros indicadores que toman en cuenta estas evaluaciones Internacionales.

En atención a lo expresado, se hace necesario realizar un estudio siendo el objetivo fundamental analizar y determinar la relación significativa entre la investigación científica con la motivación de logro académico según los estudiantes de Postgrado maestría con mención Evaluación y Acreditación de la Calidad Educativa, Investigación Educativa en la Universidad Alas Peruanas Lima, 2015.

Este trabajo de investigación nos permitirá aportar con conocimientos claros y precisos acerca de nuestra realidad en relación a la motivación para la investigación. Ya que se tiene acceso a la población y muestra y se cuenta con el tiempo y recursos necesarios para su realización

\section{MATERIALES Y MÉTODOS}

La presente investigación tiene como objetivo determinar la relación que existe entre; la investigación científica y la motivación de logro académico, tipo descriptivo y correlacional, diseño no experimental, de corte transversal. Este estudio no se limita a describir, sino que, además, nos permitirá inferir sus resultados hacia poblacio- nes, se aplicó un cuestionario que incluyó ítems de ambas variables, para ello se usó la técnica de la encuesta, el instrumento fue validado a través el alfa de cronbach para medir la confiabilidad. Se ha utilizado el análisis de relaciones utiliza las correlaciones simples entre los puntajes de las dimensiones y su respectiva variable, luego se calcula el coeficiente de correlación de Spearman para cada dimensión, por ser la adecuada para este tipo de variables (ordinales) con ello se construye el modelo estructurado ecuación modelo siendo los siguientes resultados :

La Investigación Científica se relaciona directa y significativamente a un nivel de confianza del 95\% con la motivación de logro académico en los Estudiantes de Postgrado Maestría en Evaluación y Acreditación de la Calidad Educativa, Investigación Educativa en la Universidad Alas Peruanas Lima, 2015.

La población estaba constituida por 135 estudiantes y la muestra de 57 estudiantes de Postgrado Maestría en Evaluación y Acreditación de la Calidad Educativa, Investigación Educativa en la Universidad Alas Peruanas Lima, 2015-I

Para el levantamiento de la información se solicitó autorización a la Universidad Alas Peruanas- Lima, y posteriormente se conversó con los estudiantes para solicitarles su colaboración garantizando la seguridad y anonimato de los datos recolectados.

\section{RESULTADOS}

Un aspecto que constituye un factor crítico del éxito en cualquier investigación es la validez y fiabilidad de los instrumentos de recolección de datos utilizados. Se analizó la confiabilidad del instrumento mediante la aplicación del Método de Consistencia Interna ya que el estudio se centra en la opinión de los estudiantes a los cuales se les aplicó la encuesta usando un sólo instrumento, es decir, una sola administración del instrumento de medición. Se usó el Modelo alfa (Modelo de consistencia interna de Cronbach, 1951). 
La confiabilidad, mediante el Alfa de Cronbach, indica en qué medida las diferencias individuales de los puntajes en un test pueden ser atribuidos a las diferencias "verdaderas" de las características consideradas.

Tabla $\mathrm{N}^{\circ} 1$ Distribución por género de los estudiantes de postgrado Maestría en EACE - Investigación Educativa de la Universidad Alas Peruanas Lima, 2015

$\begin{array}{llll} & \text { Frecuencia } & \text { Porcentaje } & \begin{array}{l}\text { Porcentaje } \\ \text { acumulado }\end{array} \\ \text { Femenino } & 45 & 78,90 \% & 78,90 \% \\ \text { Masculino } & 12 & 21,10 \% & 100,00 \% \\ \text { Total } & 57 & 100,00 \% & \end{array}$

El 78,9\% de los estudiantes de la Maestría en EACE - Investigación Educativa de la Universidad Alas Peruanas son mujeres

Tabla N² Variable 1: La investigación Científica

$\begin{array}{llll} & \text { Frecuencia } & \text { Porcentaje } & \begin{array}{l}\text { Porcentaje } \\ \text { acumulado }\end{array} \\ \text { Deficiente } & 2 & 3,50 \% & 3,50 \% \\ \text { Regular } & 37 & 64,90 \% & 68,40 \% \\ \text { Bueno } & 18 & 31,60 \% & 100,00 \% \\ \text { Total } & 57 & 100,00 \% & \\ & & & \end{array}$

Sobre la variable Calidad de Investigación Científica, el 64.9\% de los estudiantes señala que el nivel es regular, y un $31 \%$ que el nivel es bueno. Solo el 3.5\% opinó que era deficiente.

Tabla N³ Variable 2: Motivación de logro académico

\begin{tabular}{llll} 
& Frecuencia & Porcentaje & $\begin{array}{l}\text { Porcentaje } \\
\text { acumulado }\end{array}$ \\
Baja & 7 & $12,30 \%$ & $12,30 \%$ \\
Media & 16 & $28,10 \%$ & $40,40 \%$ \\
Alta & 34 & $59,60 \%$ & $100,00 \%$ \\
Total & 57 & $100,00 \%$ & \\
\hline
\end{tabular}

El 59.6\% de los estudiantes de Maestría en EACE - Investigación Educativa tiene un alto grado de motivación del logro académico, el 28,1\% tiene un grado medio y el 12.3\% tiene baja motivación. 
Tabla Nº Motivación Intrínseca

$\begin{array}{llll} & \text { Frecuencia } & \text { Porcentaje } & \begin{array}{l}\text { Porcentaje } \\ \text { acumulado }\end{array} \\ \text { Baja } & 7 & 12,30 \% & 12,30 \% \\ \text { Media } & 14 & 24,60 \% & 36,80 \% \\ \text { Alta } & 36 & 63,20 \% & 100,00 \% \\ \text { Total } & 57 & 100,00 \% & \\ & & & \end{array}$

Podemos apreciar que el $63.2 \%$ de los estudiantes de la maestría en Investigación Científica tiene una alta motivación intrínseca mientras que el $24.6 \%$ opina que es media. Solo un $12.3 \%$ opina que es baja.

Tabla Nº5 Motivación Extrínseca

$\begin{array}{cccc} & \text { Frecuencia } & \text { Porcentaje } & \begin{array}{c}\text { Porcentaje } \\ \text { acumulado }\end{array} \\ \text { Baja } & 8 & 14,00 \% & 14,00 \% \\ \text { Media } & 15 & 26,30 \% & 40,40 \% \\ \text { Alta } & 34 & 59,60 \% & 100,00 \% \\ \text { Total } & 57 & 100,00 \% & \\ & & & \end{array}$

Podemos apreciar que el 59.6\% de los estudiantes de la maestría en Investigación Científica tiene una alta motivación extrínseca mientras que el $26.3 \%$ opina que es media. Solo un $14 \%$ opina que es baja.

\section{DISCUSIÓN}

Los hallazgos encontrados nos demuestran la importancia de la investigación científica en la calidad de motivación de logro académico según los estudiantes de Maestría en Evaluación y Acreditación de la Calidad Educativa, Investigación Educativa en la Universidad de Alas Peruanas, evidenciándose mediante el análisis estadístico efectuado.

Del análisis descriptivo de las variables sociodemográficas se determinó que los estudiantes de Maestría en EACE Investigación Educativa, en su mayoría son mujeres (78.9\%) y que la edad promedio es de 45 años.

Al respecto Garrido, I. \& Pérez, M. (1) manifiestan que en poblaciones extranjeras muestran que las mujeres presentan una motivación de logro académico menor que los varones.

Algunos autores explican esto planteando que las mujeres se encuentran influenciadas por sutiles presiones sociales que las dirigen a metas de cuidado y afecto, mientras que los varones son dirigidos a metas de logro, éxito y poder.

Respecto a la variable Investigación Científica, encontramos que el $64.9 \%$ de los estudiantes señala que el nivel es regular, y un 31\% que el nivel es bueno. Solo el 3.5\% opinó que era deficiente. 


\section{Figura $\mathrm{N}^{\circ} 1$ : Investigación científica}

\section{V1. CALIDAD DE INVESTIGACIÖN CIENTIFICA}

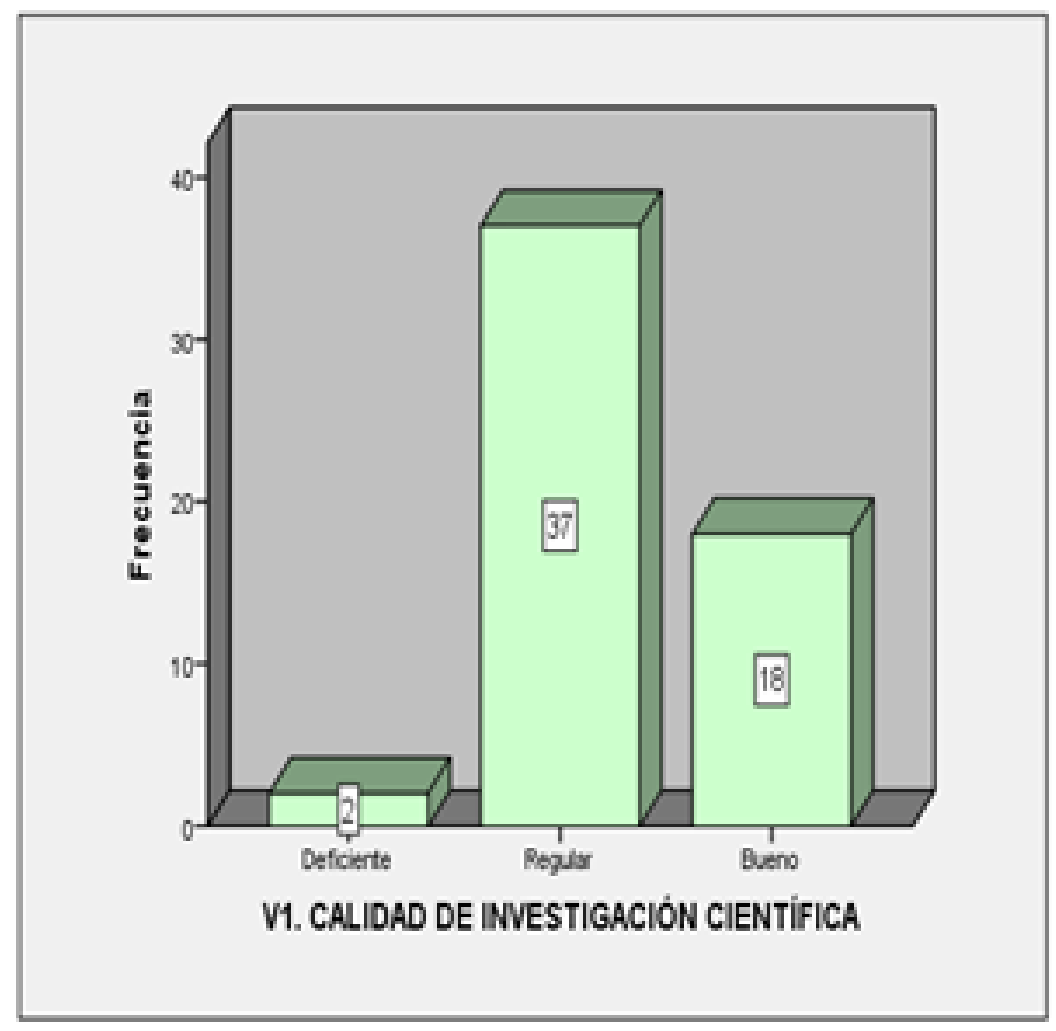

Esta distribución de resultados es alentadora, ya que el porcentaje para el nivel bueno es significativo, y para el nivel deficiente es casi nulo.

Analizando dimensionalmente la variable Investigación Científica, se observó que la mayoría de dimensiones tuvo una calificación regular o bueno por parte de los estudiantes.

Según Espinoza (2) la actitud frente a la investigación científica que más predomina entre los estudiantes es la actitud favorable, entendiéndose que están de acuerdo con hacer investigación. Entre sus conclusiones la actitud frente a la investigación científica en la dimensión cognitiva de los estudiantes es favorable en el mayor porcentaje de estudiantes.

Por tanto el artículo que antecede, se enfoca a la variable la "investigación científica", esto tiene relación con el tema que se está trabajando, además que tiene como objetivo determinar la actitud frente a la investigación científica en los estudiantes, y se pudo determinar que la conducta frente a la investigación científica es favorable.

Las dimensiones que presentaron un mayor porcentaje de calificación deficiente fueron Dominio Tecnológico Especializado (21\%) y habilidades para el uso de los procedimientos de investigación (9\%). Esto refleja una debilidad en los estudiantes dada por la falta de manejo de software especializado para investigación como el SPSS y otros en el poco dominio de una metodología para investigación.

Para Villamizar, (3) La importancia de las Tecnologías de Información ha crecido dramáticamente en las últimas décadas y probablemente seguirán creciendo. Muchos negocios están dependiendo de las Tecnologías de Información para mejorar la eficiencia y eficacia de sus operaciones, y algunos para su supervivencia. 
Para el ser humano es indispensable la tecnología, y vital en la vida diaria; para nuestra investigación se requiere de este medio y de un dominio, y en nuestras dimensiones de investigación que se ha desarrollado muchos de los docentes aun no dominan y menos para un trabajo de investigación, en casos de los programas en estadística.

En contraste, las dimensiones que presentaron un mayor porcentaje de calificación buena fueron Dominio Tecnológico Básico (77\%) y dominio para la comunicación de resultados (58\%). Esto muestra como fortaleza de los estudiantes sus conocimientos básicos en ofimática y en la comunicación y presentación de resultados de investigación.

Así mismo Izquierdo, Pardo \& Sánchez (4) manifiesta en su investigación el objetivo de analizar la incidencia de las tecnologías de la información y las comunicaciones en el proceso de formación de la investigación científica de los estudiantes universitarios lo cual permite revelar que tanto el modelo como la estrategia resultan imprescindibles en el desarrollo de los procesos de la educación superior $y$, específicamente, en el perfeccionamiento de la dinámica del proceso de formación de la investigación científica, con el empleo de estas tecnologías.

La dinámica del proceso de formación de la investigación científica sustentada en las TIC contribuye al impulso de la actividad investigativa de los estudiantes universitarios, a partir de las relaciones esenciales entre sus configuraciones y dimensiones.

Respecto a la variable Calidad de Motivación del Logro Académico, El 59.6\% de los estudiantes de Maestría en EACE - Investigación Educativa tiene un alto grado de motivación del logro académico, el $28,1 \%$ tiene un grado medio y el $12.3 \%$ tiene baja motivación.

\section{Figura 2: La motivación de logro académico}

\section{V2. MOTIVACIÓN DEL LOGRO ACADÉMICO}

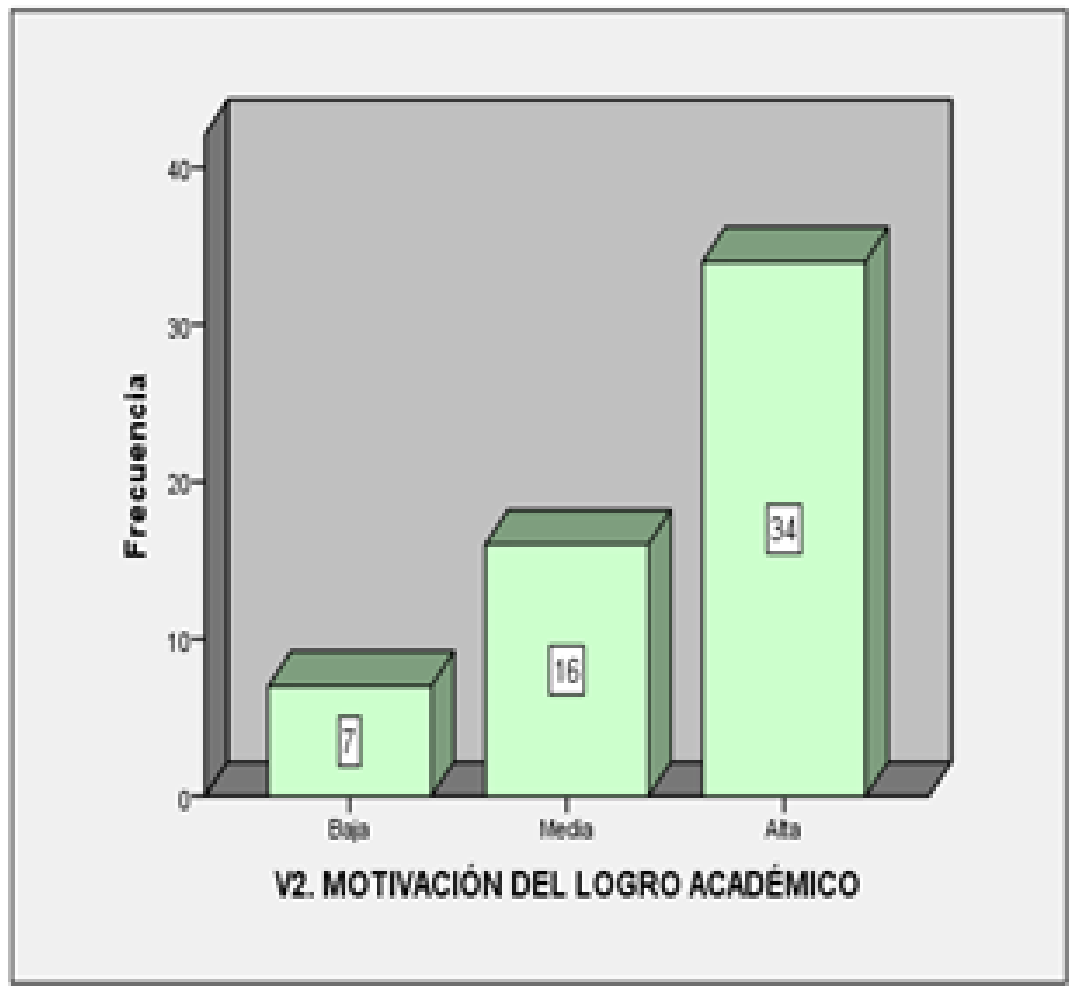


Analizando dimensionalmente la variable $\mathrm{Ca}$ lidad de Motivación del Logro Académico, se observó que en ambas dimensiones, intrínseca y extrínseca, alrededor del 60\% de los estudiantes mostró un alto nivel de motivación, siendo el porcentaje mayor en motivación intrínseca mientras que alrededor del $13 \%$ mostró baja motivación.

Para Murray, (5) el primero en definir la motivación de logro y lo hace planteando que se trata de una necesidad que lleva a una persona a hacer algo. En sus estudios Murray encontró que las personas con alta motivación de logro eran más perseverantes.

Con las pruebas de hipótesis se verificó la relación que existe entre la investigación científica y la motivación del logro académico, así como la relación que hay entre las dimensiones de la 1ra variable y la 2 da variable.

En la prueba de hipótesis general se determinó con un nivel de confianza del 95\% que la relación entre Investigación Científica y la Motivación del Logro Académico es significativa y directa con un valor de 0.840 en una escala de 0 a 1 estimado con el coeficiente Gamma. Esto se fundamenta en que la buena calidad de investigación científica, contribuye a una mayor motivación del logro académico.

\section{REFERENCIAS BIBLIOGRÁFICAS}

1. Garrido G. I. (1996). Psicología de la motivación. Madrid: Síntesis, 195-232.

2. Espinoza D. (2015). "Actitud frente a la investigación científica en los estudiantes del 7mo ciclo de la Escuela Académico Profesional de Enfermería de la Universidad Alas Peruanas, 2010". Lima- Perú. Disponible en: http:// repositorio.uap.edu.pe/handle/uap/2934.

3. Villamizar L. (2008). Uso e importancia de las tecnologías de información y comunicaciones (tics) en la docencia universitaria: un estudio de caso utilizando let me learn Revista colombiana de Tecnologias Avanzadas. 9296. Disponible en : http://www.unipamplona.edu.co/unipamplona/portalIG/home_40/recursos/02_v07_12/revista_11/16112011/v11_14.pdf
4. Izquierdo J.; Pardo M. \& Sánchez L. (2010). “El proceso de formación para la investigación científica en la educación superior sustentado en las tecnologías de la información y las comunicaciones". Santiago de Cuba-Cuba. Disponible en: motivación de logro. Psicothema, 333-351.

5. Murray H. (1938). Exploration in Personaty. New York: Oxford Universaty Press. 